\title{
BER Analysis of Joint Network/Channel decoding in Block Rayleigh fading channels
}

\author{
Xuan-Thang Vu, Marco Di Renzo and Pierre Duhamel \\ Laboratory of Signals and Systems (L2S), French National Center for Scientific Research (CNRS) - \\ École Supérieure d'Électricité (SUPÉLEC) - University of Paris-Sud 11, \\ 3 rue Joliot-Curie, 91192 Gif-sur-Yvette (Paris), France, \\ E-Mail: \{xuanthang.vu, marco.direnzo, pierre.duhamel\}@1ss.supelec.fr.
}

\begin{abstract}
This paper studies the four-node Multiple Access Relay Channel (MARC) under quasi-static block Rayleigh fading channels and Gaussian noise environment. A relay employs Demodulate-and-Forward (DMF) protocol in order to help two channel-encoded sources to communicate with a destination. The contributions of the paper are threefold: i) we propose a Near Optimal Joint Network/Channel decoding (NO-JNCD) algorithm at the destination. The NO-JNCD employs Cooperative Maximum Ratio Combining (C-MRC) detector and the BCJR algorithm applied to a compound code which trellis consists of all possible states of two single trellises at sources; ii) we compute extended distance spectrum of the compound code containing input weights for each source and output weights on each fading channel; and iii) from the extended distance spectrum, we derive the Bit Error Rate (BER) upper bound for the compound code as well as for each source. It is shown by simulation that the proposed decoder provides performance very close to that of the optimal JNCD with both DMF and Decode-and-Forward (DF) relaying protocols. Finally, the analysis is checked by simulation.
\end{abstract}

Keywords-Joint Network/Channel decoding, Multiple Access Relay Channels, Convolutional Code, Distance Spectrum.

\section{INTRODUCTION}

Network Coding (NC), in which intermediate nodes are allowed to forward a linear combination of multiple input packets [1] has gained much attention because of its potential improvement in diversity gain and throughput over classical routing techniques. It has been recently shown that $\mathrm{NC}$ can also provide improved performance and energy efficiency compared with conventional network routing techniques [2]. However, $\mathrm{NC}$ is facing a fundamental error propagation problem [3] which can dramatically degrade performance and reduce the diversity order of cooperative networks.

Joint network channel decoding (JNCD) has gained much attention [4] as an effective solution to counteract the error propagation problem [2]. The idea behind JNCD is the exploitation of the inherent redundancy of network and channel codes. It has been shown in [4] that, compared to conventional distributed turbo coding and separate network and channel decoding, JNCD can improve the performance of canonical two-way and multiple-access relay channels. However, most results assume that only correct packets are forwarded from the relay to the destination. Other JNCD algorithms were also proposed for MARC with both Convolutional Code (CC) and Low Density Parity Check (LDPC) code [5], [6]. Likewise, it was assumed that the source-relay channels are error-free. Recently, various relaying protocols have addressed the error propagation problem in cooperative communications, including soft relaying protocols [7], the threshold-based relaying [8] and opportunistic relaying [9]. Other solutions foresee that the destination takes care of error propagation. The idea is that, if the destination has access to the channel state information (CSI) of the source-relay links, this knowledge can be exploited to mitigate the error propagation problem. The authors in [10] show that channel-aware receivers can significantly improve the performance of NC. However, no channel coding is considered in [10]. A cooperative communication scheme for multiple-input multiple-output (MIMO) was proposed in [8]. Note that these papers mostly provided simulations but did not provide analytical results because of the difficulty of analyzing iterative decoding [4] or LDPC codes [6].

Therefore, performance analysis in terms Bit Error Rate (BER) is important in order to understand and efficiently design a cooperative system. This paper studies the four-node multiple access Demodulate-and-Forward (DMF) [11] relay network under quasi-static block Rayleigh fading channels. In particular, we propose a near optimal JNCD (NO-JNCD) algorithm at the destination which simultaneously decodes the data messages. A Cooperative Maximal Ratio Combining (CMRC) detector [12] is employed prior to channel decoding in order to tackle with the error propagation and to reduce the decoder's complexity. The key idea behind the proposed algorithm is that we consider the compound code at destination with a trellis consisting of all states of single trellis at sources [13]. Hence, the relayed signal can be seen as an additional redundancy inside the compound code. The idea of using the combined trellis can also be found in [14] for the two way relay network and physical network coding. However, no analytical analysis was available in [14] and the combined code was only used to decode network-coded symbols at the relay.

After describing the system model in Section II, and presenting the proposed NO-JNCD algorithm in Section III the contributions of the paper are as follows: i) First, we derive distance spectrum and the minimum distance of the compound code in Section IV. We show that the output weights of the compound codeword always distribute on two or three orthogonal channels; ii) From this distance spectrum, we derive in Section V the upper bound of BER for each source in block Rayleigh fading channels. In addition, the proposed NO-JNCD algorithm is also compared by simulation to the optimal JNCD (OJNCD) algorithm [13] in both Decode-and-Forward (DF) and DMF protocols. The simulations provided in Section VI show that NO-JNCD provides a performance very close to that 
obtained by the OJNCD algorithm in both relaying protocols.

\section{SySTEM MODEL}

The network under consideration consists of two sources $\left(\mathrm{S}_{1}, \mathrm{~S}_{2}\right)$, one destination (D) and one relay $(\mathrm{R})$. All nodes are equipped with single antennas. The system is assumed to operate on orthogonal channels. Therefore, a cooperation period is divided into two phases: broadcasting phase and relaying phase. In the first phase, each source broadcasts its messages to $\mathrm{R}$ and $\mathrm{D}$. In the second phase, the relay forwards network-encoded symbols to the destination. All channels are subject to quasi-static block Rayleigh fading plus Additive White Gaussian Noise (AWGN) [15]. The relay employs DMF protocol [11]. More specifically, source $\mathrm{S}_{i}, i=1,2$ encodes a message $\mathbf{u}_{i}$ consisting of $K$ data symbols into a codeword $\mathbf{c}_{i}$, which contains $N K$ coded symbols by a convolutional code (CC) of rate of $1 / N, \mathbf{c}_{i}=\left\{c_{i, 1}, \ldots, c_{i, N K}\right\}$. The codeword $\mathbf{c}_{i}$ is then binary mapped into a signal $\mathbf{x}_{i}$ as $x_{i, k}=2 c_{i, k}-1, k=\{1, \ldots, N K\}$, where $x_{i, k}$ is the $k$ th symbol of $\mathbf{x}_{i}$, before being transmitted to the relay and the destination. The received signals at the destination and the relay at the end of first phase are given as follows:

$$
\begin{aligned}
& \mathbf{y}_{S_{i} D}=\sqrt{P_{S_{i} D}} h_{S_{i} D} \mathbf{x}_{i}+\mathbf{n}_{i D}, i=1,2 \\
& \mathbf{y}_{S_{i} R}=\sqrt{P_{S_{i} R}} h_{S_{i} R} \mathbf{x}_{i}+\mathbf{n}_{i R}, i=1,2
\end{aligned}
$$

where $P_{X Y}$ with $X \in\left\{S_{1}, S_{2}\right\}$ and $Y \in\{D, R\}$ is the power of the received signal at node $\mathrm{Y}$ from node $\mathrm{X}$ (which contains the pathloss); $h_{X Y}$ is the channel coefficient of link X-Y, which is a complex Gaussian random variable with zero mean, unit variance, $\mathbb{E}\left\{\left|h_{X Y}\right|^{2}\right\}=1$ and is statistically independent among different $\mathrm{X}-\mathrm{Y}$ channels; $\mathbf{n}_{X Y}$ is a noise vector whose components are Gaussian random variables with zero mean and variance $\sigma^{2}$.

At the end of the first phase, the relay: i) estimates a codeword $\hat{\mathbf{c}}_{i}=\left\{\hat{c}_{i, k}\right\}_{k=1}^{N K}$ by a Maximum Likelihood (ML) detector: $\hat{c}_{i, k}=\arg \max _{c_{i, k} \in\{0,1\}}\left\{\left|y_{S_{i} R, k}-\sqrt{P_{S_{i} R}} h_{S_{i} R} x_{i, k}\right|^{2}\right\}$; ii) network-encodes $\hat{\mathbf{c}}_{1}, \hat{\mathbf{c}}_{2}$ to get binary network-encoded signal $\hat{\mathbf{c}}_{R}$, with $\hat{c}_{R, k}=\hat{c}_{1, k} \oplus \hat{c}_{2, k}$; iii) modulates $\hat{\mathbf{c}}_{R}$ into signal symbols $\hat{\mathbf{x}}_{R}$ and forwards it to the destination regardless the possible decoding error at the relay. The received signal at the destination from relay is given by

$$
\mathbf{y}_{R D}=\sqrt{P_{R D}} h_{R D} \hat{\mathbf{x}}_{R}+\mathbf{n}_{R D} .
$$

The destination is assumed to be aware of source-relay channels. After two frames, the destination begins decoding in two steps: i) applies the C-MRC decoder [12] and ii) performs joint network channel decoding.

\section{NeAR Optimal Joint Network Channel DECODING}

After receiving three channel observations from two sources and from the relay, the destination performs joint network/channel decoding based on the knowledge of the source-relay channel gains. The MAP decision rule is given by

$$
\begin{aligned}
{\left[\hat{u}_{1, k}, \hat{u}_{2, k}\right] } & =\arg \max _{u_{1, k}, u_{2, k}} \operatorname{Pr}\left\{u_{1, k}, u_{2, k} \mid \mathbf{y}_{S_{1} D}, \mathbf{y}_{S_{2} D}, \mathbf{y}_{R D}\right\} \\
& \sim \arg \max _{u_{1, k}, u_{2, k}} \operatorname{Pr}\left\{u_{1, k}, u_{2, k}, \mathbf{y}_{S_{1} D}, \mathbf{y}_{S_{2} D}, \mathbf{y}_{R D}\right\}
\end{aligned}
$$

In order to make full use of the redundancy provided by the relay, the destination jointly decodes both data messages $\mathbf{u}_{1}, \mathbf{u}_{2}$ simultaneously. The key point of our study is to consider the trellis at the destination as a compound trellis that contains all possible combinations of the individual trellis states at $\mathrm{S}_{1}, \mathrm{~S}_{2}$ and the relayed signal provides additional parity bits (redundancy) to the compound code. Let $\mathrm{g}$ be the generator polynomial of channel code at two sources, the generator polynomial of the compound code at destination, $\mathbf{G}$, is:

$$
\mathbf{G}=\left[\begin{array}{lll}
\mathbf{g} & \mathbf{g} & 0 \\
\mathrm{~g} & 0 & \mathrm{~g}
\end{array}\right]
$$

Now the problem in (3) can optimally be solved by using the BCJR algorithm [16] for the compound code $\mathbf{G}$, with compound data message $\mathbf{U}=\left\{U_{k}\right\}_{k=1}^{K}, U_{k} \triangleq\left[u_{1, k}, u_{2, k}\right]$ and compound codeword $\mathbf{C}=\left\{C_{k}\right\}_{k=1}^{N K}, C_{k} \triangleq\left[c_{1, k}, c_{2, k}, c_{R, k}=\right.$ $\left.c_{1, k} \oplus c_{2, k}\right]$. Denote $S_{1}^{k}, S_{2}^{k}$ as the states of trellis in $\mathrm{S}_{1}$ and $\mathrm{S}_{2}$ at time index $k ; \alpha_{k}\left(S_{1}^{k}, S_{2}^{k}\right), \beta_{k}\left(S_{1}^{k}, S_{2}^{k}\right)$ and $\chi_{k}\left(S_{1}^{k}, S_{2}^{k}, S_{1}^{k+1}, S_{2}^{k+1}\right)$ as forward filter, backward filter and metric of the compound code. The forward and backward filters can be recursively computed from the compound metric [16] which is:

$$
\begin{aligned}
& \chi_{k}\left(S_{1}^{k}, S_{2}^{k}, S_{1}^{k+1}, S_{2}^{k+1}\right)=\sum_{i=N k+1}^{N(k+1)} \\
& \left(\begin{array}{l}
\left|y_{1, i}-\sqrt{P_{S_{1} D}} h_{S_{1} D} x_{1, i}\right|^{2}+\left|y_{2, i}-\sqrt{P_{S_{2} D}} h_{S_{2} D} x_{2, i}\right|^{2} \\
+\lambda_{R}\left|y_{R, i}-\sqrt{P_{R D}} h_{R D} \hat{x}_{R, i}\right|^{2}
\end{array}\right),
\end{aligned}
$$

where $\lambda_{R}=\frac{\min \left\{\gamma_{S_{1} R}, \gamma_{S_{2} R}, \gamma_{R D}\right\}}{\gamma_{R D}}$ is the parameter of C-MRC detector [12] that takes into account decoding error at the relay. Here, $\gamma_{X Y}=P_{X Y}\left|h_{X Y}\right|^{2} / \sigma^{2}$ with $X \in\left\{S_{1}, S_{2}, R\right\}$ and $Y \in\{R, D\}$ denotes the instantaneous SNR of link X-Y.

In (5), the proposed decoder considers relayed signal as additional redundancy of compound code. The NC and CC are optimally decoded in one step. The compound trellis of (4) is same as that of the OJNCD algorithm [13] and its complexity doubly exponentially increases with constraint length of the single code g. Unlike [13], in which the decoder examines all possible values of estimated bits at the relay, our solution assumes that relayed bits are equivalent to correct bits transmitted via some equivalent channels. Although being more complex than iterative decoding algorithm, the proposed algorithm allows to analyze the system performance.

\section{Distance Spectrum of the Compound Code}

It is well known that the performance of convolutional code depends on its distance spectrum - distribution of input weights and coded (output) weights of non-zero paths [17]. In our case, since the output of the compound code propagates via three blocks, i.e. $h_{S_{1} D}, h_{S_{2} D}$ and $h_{R D}$, we derive an extended distance spectrum of the compound code, which facilitates performance analysis in the next section, as follows:

$$
\Gamma(W, D)=\sum_{\substack{w_{1}+w_{2}=w \\ d_{1}+d_{2}+d_{3}=d}} \Gamma_{w_{1} w_{2} d_{1} d_{2} d_{3}} W_{1}^{w_{1}} W_{2}^{w_{2}} D_{1}^{d_{1}} D_{2}^{d_{2}} D_{3}^{d_{3}}
$$

where $W_{1}, W_{2}$ are placeholders for inputs $\mathbf{u}_{1}, \mathbf{u}_{2} ; D_{1}, D_{2}, D_{3}$ are placeholders for coded outputs propagating via channel 
$h_{S_{1} D}, h_{S_{2} D}$ and $h_{R D}$, respectively; $w_{1}, w_{2}$ are respective data input weights of $\mathbf{u}_{1}, \mathbf{u}_{2} ; d_{1}, d_{2}, d_{3}$ are coded output weights of channels $h_{S_{1} D}, h_{S_{2} D}$ and $h_{R D}$; and $\Gamma_{w_{1} w_{2} d_{1} d_{2} d_{3}}$ is the number of paths with parameters $w_{1}, w_{2}, d_{1}, d_{2}, d_{3}$. For example, $2 W_{1}^{2} W_{2} D_{1} D_{2}^{3} D_{3}^{2}$ shows that there are $\Gamma_{21132}=2$ non-zero paths with input weight 3 including weight 2 for $\mathbf{u}_{1}$ and weight 2 for $\mathbf{u}_{2}$; output weight 6 consisting of weight 1 for channel $h_{S_{1} D}$, weight 3 for channel $h_{S_{2} D}$ and weight 2 for channel $h_{R D}$. Computation of the distance spectrum in (6) is easily obtained via the well-known algorithm in [18]. Table I shows an example of extended distance spectrum in (6) for $d=14$.

Define $\mathbf{D}_{d} \triangleq\left\{d_{1}, d_{2}, d_{3}\right\}$ with $d_{1}+d_{2}+d_{3}=d$ as a weight pattern corresponding to weight $d$. Obviously, the weight pattern $\mathbf{D}_{d}$ defines the distribution of weights among the three channels.

TABLE I. EXtended Distance SPECTRUM OF $\mathbf{G}$ IN (4), $\mathbf{g}=[23,35]$

\begin{tabular}{cccccc}
\hline \hline$\Gamma_{w_{1} w_{2} d_{1} d_{2} d_{3}}$ & $w_{1}$ & $w_{2}$ & $d_{1}$ & $d_{2}$ & $d_{3}$ \\
\hline 1 & 0 & 1 & 0 & 7 & 7 \\
1 & 0 & 3 & 0 & 7 & 7 \\
1 & 1 & 0 & 7 & 0 & 7 \\
1 & 3 & 0 & 7 & 0 & 7 \\
1 & 1 & 1 & 7 & 7 & 0 \\
1 & 3 & 3 & 7 & 7 & 0 \\
\hline
\end{tabular}

Lemma 1. Denote $f$ as the minimum distance of the individual codes at sources $\mathrm{S}_{1}$ and $\mathrm{S}_{2}$. The minimum distance $F$ of the compound code is equal to twice the minimum distance of the single code, $F=2 f$, and the weight pattern $\mathbf{D}_{F}$ has one of the following values $\{f, f, 0\},\{f, 0, f\},\{0, f, f\}$.

Proof: Denote $\mathcal{H}(\mathbf{x})$ as weight of vector $\mathbf{x}$, i.e. number of non-zeros bits. Let $\tilde{\mathbf{C}}=\left[\tilde{\mathbf{c}}_{1}, \tilde{\mathbf{c}}_{2}, \tilde{\mathbf{c}}_{R}\right]$ be a compound codeword with weight $F$, where $\tilde{\mathbf{c}}_{1}, \tilde{\mathbf{c}}_{2}$ is a codeword at source $\mathrm{S}_{1}$ and $\mathrm{S}_{2}$, and $\tilde{\mathbf{c}}_{R}=\left\{\tilde{c}_{R, k}\right\}_{k=1}^{N K}$ with $\tilde{c}_{R, k}=\tilde{c}_{1, k} \oplus \tilde{c}_{2, k}$. Let $d_{1}=$ $\mathcal{H}\left(\tilde{\mathbf{c}}_{1}\right), d_{2}=\mathcal{H}\left(\tilde{\mathbf{c}}_{2}\right), d_{3}=\mathcal{H}\left(\tilde{\mathbf{c}}_{R}\right)$. Obviously, $\mathcal{H}(\tilde{\mathbf{C}})=d_{1}+$ $d_{2}+d_{3}=F$. Because $F$ is the minimum distance of compound code, $\tilde{\mathbf{c}}_{1}, \tilde{\mathbf{c}}_{2}$ is either all-zero codeword or minimum weight codeword, i.e. $d_{1}, d_{2} \in\{0, f\}$. If $d_{1}=f, d_{2}=0$, it is to see that $d_{3}=d_{1}=f$, leading to $F=2 f$ and $\mathbf{D}_{F}=\{f, 0, f\}$. If $d_{1}=0, d_{2}=f$, then $d_{3}=d_{2}=f$. Similarly we have $F=2 f$ and $\mathbf{D}_{F}=\{0, f, f\}$. Now consider the case $d_{1}=f, d_{2}=f$. If $\tilde{\mathbf{c}}_{1} \equiv \tilde{\mathbf{c}}_{2}$, we have $\tilde{\mathbf{c}}_{R}=\mathbf{0}$, consequently $F=2 f$ and $\mathbf{D}_{F}=$ $\{0, f, f\}$. Otherwise, if $\tilde{\mathbf{c}}_{1} \neq \tilde{\mathbf{c}}_{2}$, there are at least two nonzero bits in $\tilde{\mathbf{c}}_{R}$, leading to $d_{3} \geqslant 2$ and $d_{1}+d_{2}+d_{3} \geqslant 2 f+2$. It means that $\tilde{\mathbf{c}}_{1} \neq \tilde{\mathbf{c}}_{2}$ does not belong to the minimum weight compound codeword $\tilde{\mathbf{C}}$.

Lemma 2. Consider the weight pattern $\mathbf{D}_{d}=\left\{d_{1}, d_{2}, d_{3}\right\}$ of the compound codeword $\mathbf{X}$ with weight $d>F$. There are at least two non-zero elements in $\mathbf{D}_{d}$.

Proof: Let $\mathbf{C}=\left[\mathbf{c}_{1}, \mathbf{c}_{2}, \mathbf{c}_{R}\right]$ be a compound codeword with weight $d>F$. Obviously, there is at least one non-zero bit in $\mathbf{c}_{1}, \mathbf{c}_{2}$. Without loss of generality, assuming that $c_{1,1}=1$. If $c_{2,1}=0$, then $c_{R, 1}=1$ and there is at least one weight in channel $h_{S_{1} D}$ and $h_{R D}$. If $c_{2,1}=1$, then there is at least one weight in $h_{S_{1} D}$ and $h_{R D}$. This proves Lemma 2.

Lemma 1 and Lemma 2 provide an important information that the output weights of the compound code always locate on two or three orthogonal channels. This is used along with input weights in (6) to evaluate the performance of NO-JNCD in next section.

\section{PERFormanCE ANALYSis}

\section{A. Equivalent channel of network-coded symbols}

It is well known that the two-hop channel of the networkcoded symbols can be tightly modeled as a single-hop channel $\gamma_{R}=\min \left\{\gamma_{S_{1} R}, \gamma_{S_{2} R}, \gamma_{R D}\right\}$ [12], where $\gamma_{X Y}=\frac{P_{X Y}}{\sigma^{2}}\left|h_{X Y}\right|^{2}$ is instantaneous SNR of X-Y channel with average SNR $\bar{\gamma}_{X Y}=\frac{P_{X Y}}{\sigma^{2}}$. Because $\gamma_{X Y}$ is exponentially distributed, it is easy to see that $\gamma_{R}$ is also an exponential random variable with parameter $\bar{\gamma}_{R}$ given by

$$
\frac{1}{\bar{\gamma}_{R}}=\frac{1}{\bar{\gamma}_{S_{1} R}}+\frac{1}{\bar{\gamma}_{S_{2} R}}+\frac{1}{\bar{\gamma}_{R D}} .
$$

Using the equivalent channel, the network-coded signal is modeled as if it was conveyed by a single channel whose instantaneous SNR is $\gamma_{R}$. For convenience, define $h_{R}=$ $\sqrt{\frac{\gamma_{R} \sigma^{2}}{P_{R D}}}$ as the equivalent channel coefficient of this channel, the received signal at destination from relay is given by

$$
\mathbf{y}_{R D}=\sqrt{P_{R D}} h_{R} \mathbf{x}_{R}+\mathbf{n}_{R D},
$$

where $x_{R, k}=2\left(c_{1, k} \oplus c_{2, k}\right)-1$. Denote $\mathbf{X}=\left[\mathbf{x}_{1}, \mathbf{x}_{2}, \mathbf{x}_{R}\right]$ as the compound codeword signal. The metric of a compound codeword $\mathbf{X}$ is given as follows:

$$
\begin{gathered}
\mathbb{M}(\mathbf{X})=\sum_{k=1}^{N K}\left(\left|y_{S_{1} D, k}-\sqrt{P_{S_{1} D}} h_{S_{1} D} x_{1, k}\right|^{2}+\right. \\
\left.\left|y_{S_{2} D, k}-\sqrt{P_{S_{2} D}} h_{S_{2} D} x_{2, k}\right|^{2}+\left|y_{R, k}-\sqrt{P_{R D}} h_{R} x_{R, k}\right|^{2}\right) .
\end{gathered}
$$

We note that possible errors at the relay are taken into account by the equivalent channel $h_{R}$.

\section{B. Derivation of $B E R$}

The received signal at destination for the compound code is thus equivalent to the output of some block fading channel [15] with three blocks $h_{S_{1} D}, h_{S_{2} D}$ and $h_{R D}$ for which the BER is evaluated as [17]:

$$
\mathrm{P}_{e}=\frac{1}{2} \sum_{d=F}^{+\infty} \sum_{\mathbf{D}_{d}} \mathrm{w}\left(\mathbf{D}_{d}\right) \mathrm{P}_{u}\left(d \mid \mathbf{D}_{d}\right),
$$

where $F$ is the minimum distance of the compound code; $\mathrm{w}\left(\mathbf{D}_{d}\right)=\Gamma_{w_{1}, w_{2}, d_{1}, d_{2}, d_{3}}\left(w_{1}+w_{2}\right)$ is total data input weights associated to pattern $\mathbf{D}_{d}$; and $\mathrm{P}_{u}\left(d \mid \mathbf{D}_{d}\right)$ is pair-wise error probability (UPEP), the probability of receiving a codeword with weight $d$ when all-zero codeword was transmitted and $d$ weights are allocated in three channels $h_{S_{1} D}, h_{S_{2} D}, h_{R}$ according to pattern $\mathbf{D}_{d}$. The UPEP is averaged over channels of the conditioned pair-wise error probability (CPEP) $\mathrm{P}_{c}\left(d \mid \mathbf{D}_{d}\right)$ as follows:

$$
\mathrm{P}_{u}\left(d \mid \mathbf{D}_{d}\right)=\mathbb{E}\left\{\mathrm{P}_{c}\left(d \mid \mathbf{D}_{d}\right)\right\},
$$

where $\mathbb{E}\{$.$\} denotes the expectation over fading coefficients.$

Lemma 3. The CPEP $\mathrm{P}_{c}\left(d \mid \mathbf{D}_{d}=\left\{d_{1}, d_{2}, d_{3}\right\}\right)$ is given by

$$
\mathrm{P}_{c}\left(d \mid \mathbf{D}_{d}\right)=Q\left(\sqrt{2\left(d_{1} \gamma_{S_{1} D}+d_{2} \gamma_{S_{2} D}+d_{3} \gamma_{R}\right)}\right),
$$


where $Q(x)=\frac{1}{\pi} \int_{0}^{+\infty} e^{-\frac{t^{2}}{2}} d t$ denotes the $Q$-function.

Proof: The CPEP $\mathrm{P}_{c}\left(d \mid \mathbf{D}_{d}\right)$ is equal to the probability of receiving codeword $\mathbf{X}$ with weight $d$ when the all zero codeword $\mathbf{X}_{0}$ was transmitted:

$$
\mathbf{P}_{c}\left(d \mid \mathbf{D}_{d}\right)=\operatorname{Pr}\left\{\mathbb{M}(\mathbf{X})-\mathbb{M}\left(\mathbf{X}_{0}\right)<0 \mid \mathbf{D}_{d}\right\} .
$$

Since $\mathbf{X}$ has weight $d$, there are only $d$ non-zero elements in $\mathbb{M}(\mathbf{X})-\mathbb{M}\left(\mathbf{X}_{0}\right)$. In addition, given pattern $\mathbf{D}_{d}=\left\{d_{1}, d_{2}, d_{3}\right\}$, there are $d_{1}$ weights in channel $h_{S_{1} D}, d_{2}$ weights in channel $h_{S_{2} D}$ and $d_{3}$ weights in channel $h_{R}$. Following the method in [15] we obtain (12).

Theorem 1. The UPEP $\mathrm{P}_{u}\left(F \mid \mathbf{D}_{F}\right)$ according to the minimum distance $F$ of the compound code is:

$$
\mathrm{P}_{u}\left(F \mid \mathbf{D}_{F}\right)=\frac{3}{4 F^{2}} \frac{1}{\bar{\gamma}_{X} \bar{\gamma}_{Y}},
$$

with $X, Y \in\left\{S_{1} D, S_{2} D, R\right\}, X \neq Y$.

Proof: Lemma 1 states that pattern $\mathbf{D}_{F}$ can only take one of the values $\{f, f, 0\},\{f, 0, f\},\{0, f, f\}$. Consider the first case and apply Lemma 3:

$$
\mathrm{P}_{c}(F \mid\{f, f, 0\})=Q\left(\sqrt{2 f \gamma_{S_{1} D}+2 f \gamma_{S_{2} D}}\right) .
$$

Using alternative expression of the Q-function and integrating over the distribution of $\gamma_{S_{1} D}, \gamma_{S_{2} D}$ we obtain (15), after second-order approximation by Taylor series. Applying the same strategy for the two other cases $\{f, 0, f\},\{0, f, f\}$ results in (13).

Theorem 2. The UPEP $\mathrm{P}_{u}\left(d>F \mid \mathbf{D}_{d}=\left\{d_{1}, d_{2}, d_{3}\right\}\right)$ of the compound code is given by

$$
\mathrm{P}_{u}\left(d \mid \mathbf{D}_{d}\right)=\left\{\begin{array}{l}
\frac{3}{16 d_{i_{X}} d_{i_{Y}}} \frac{1}{\bar{\gamma}_{X} \bar{\gamma}_{Y}}, \text { if } \operatorname{sgn}\left(\mathbf{D}_{d}\right)=2 \\
\frac{5}{32 d_{1} d_{2} d_{3}} \frac{1}{\bar{\gamma}_{S_{1} D} \bar{\gamma}_{S_{2} D} \bar{\gamma}_{R}}, \text { if } \operatorname{sgn}\left(\mathbf{D}_{d}\right)=3
\end{array},\right.
$$

where $i_{X} \neq i_{Y} \in\{1,2,3\}$, $\operatorname{sgn}($.$) denotes the sign function$ and $\operatorname{sgn}\left(\mathbf{D}_{d}\right)=\operatorname{sgn}\left(d_{1}\right)+\operatorname{sgn}\left(d_{2}\right)+\operatorname{sgn}\left(d_{3}\right)$.

Proof: Lemma 2 states that the weight pattern $\mathbf{W}_{d}=$ $\left\{d_{1}, d_{2}, d_{3}\right\}$ is always distributed on two channels, i.e. $\operatorname{sgn}\left(\mathbf{D}_{d}\right)=2$, or three channels, i.e. $\operatorname{sgn}\left(\mathbf{D}_{d}\right)=3$. In the former, the same demonstration as for Theorem 1 holds. For the latter, using the CPEP given in Lemma 3 and integrating CPEP over fading channels we have (16), with

$$
I(a, b, c)=\frac{a^{2}}{(a-b)(a-c)} \sqrt{\frac{a}{a+1}},
$$

and $(*)$ results from a third-order approximation using Taylor series. This proves Theorem 2.

Theorem 1 and Theorem 2 are used along with (10) to derive the BER of the compound code, i.e. the average BER of two sources. The BER of each source is computed by considering information weights of each source in the extended distance spectrum in (6). Denote $\mathrm{w}_{i}\left(\mathbf{D}_{d}\right)=w_{i} \Gamma_{w_{1} w_{2} d_{1} d_{2} d_{3}}$ as the information weights of source $\mathrm{S}_{i}$ caused by pattern $\mathbf{D}_{d}$. The BER of each source is given as follows:

$$
\mathrm{P}_{e}\left(\mathrm{~S}_{i}\right)=\sum_{d=F}^{+\infty} \sum_{\mathbf{D}_{d}} \mathrm{w}_{i}\left(\mathbf{D}_{d}\right) \mathbf{P}_{u}\left(d \mid \mathbf{D}_{d}\right), i=1,2 .
$$

In (18), the BER of each source is a combination of diversity order 2 and diversity order 3 factors weighted by $\mathrm{w}_{i}\left(\mathbf{D}_{d}\right)$ which is strictly defined in G. At high SNR region, the BER is dominated by second-order factors. Consequently, the system achieves second-order diversity. We note that the infinity of weight $d$ in (18) is primitively derived for Gaussian channels because the Q-function decays at much higher rate than the increase rate of input weights $\mathrm{w}_{i}\left(\mathbf{D}_{d}\right)$ [17]. However, in block Rayleigh fading channel, the maximum number of $d$ in (10) is usually limited by the first values [19].

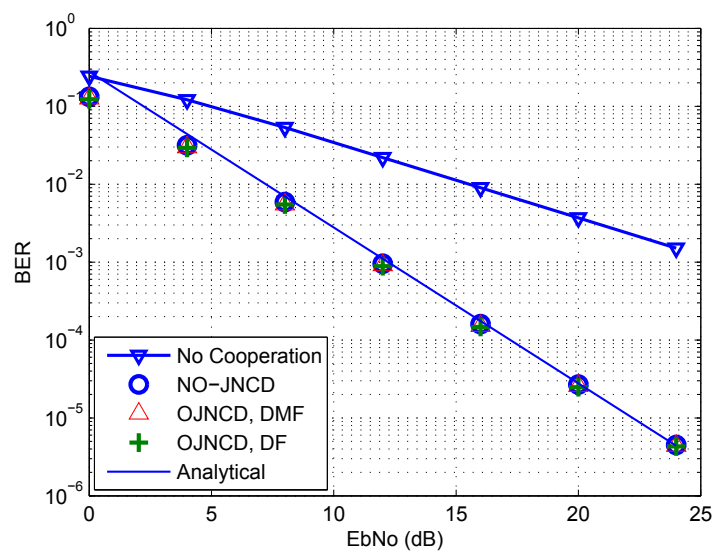

Fig. 1. Performance of NO-JNCD compared with OJNCD in both DF and DMF in Symmetric setting. The BER of two sources are the same.

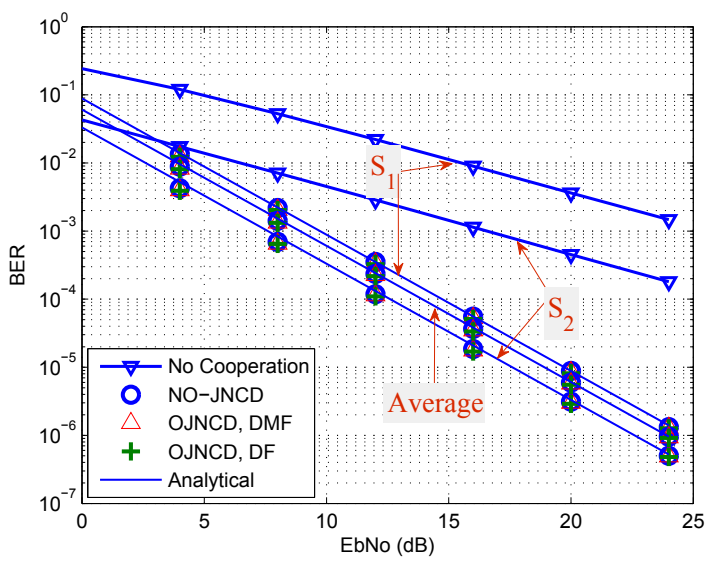

Fig. 2. Performance of NO-JNCD compared with OJNCD in both DF and DMF in Asymmetric setting.

\section{Numerical AND Simulation Results}

The system under study consists of two sources, one relay and one destination. Due to space limitation, we only consider quasi-static block Rayleigh fading channels in this paper as a special block fading scenario. The results can easily be extended to general block fading channels and will be reported elsewhere. In addition, quasi-static block fading case makes 


$$
\begin{aligned}
& \mathrm{P}_{u}(F \mid\{f, f, 0\})=\int_{0}^{+\infty} \int_{0}^{+\infty}\left(\frac{1}{\pi} \int_{0}^{\pi / 2} e^{-\frac{f \gamma_{S_{1} D}+f \gamma_{S_{2} D}}{\sin ^{2} \theta}} d \theta\right) \frac{1}{\bar{\gamma}_{S_{1} D} \bar{\gamma}_{S_{2} D}} e^{-\frac{\gamma_{S_{1} D}}{\bar{\gamma}_{S_{1} D}}-\frac{\gamma_{S_{2} D}}{\bar{\gamma}_{S_{2} D}}} d \gamma_{S_{1} D} d \gamma_{S_{2} D} \\
& =\frac{1}{2}\left(1-\frac{\bar{\gamma}_{S_{1} D}}{\bar{\gamma}_{S_{1} D}-\bar{\gamma}_{S_{2} D}} \sqrt{\frac{f \bar{\gamma}_{S_{1} D}}{1+f \bar{\gamma}_{S_{1} D}}}-\frac{\bar{\gamma}_{S_{2} D}}{\bar{\gamma}_{S_{2} D}-\bar{\gamma}_{S_{2} D}} \sqrt{\frac{f \bar{\gamma}_{S_{2} D}}{1+f \bar{\gamma}_{S_{2} D}}}\right) \simeq \frac{3}{16 f^{2}} \frac{1}{\bar{\gamma}_{S_{1} D} \bar{\gamma}_{S_{2} D}} \\
& \mathrm{P}_{u}\left(d \mid\left\{d_{1}, d_{2}, d_{3}\right\}\right)=\int_{0}^{+\infty} \int_{0}^{+\infty} \int_{0}^{+\infty}\left(\frac{1}{\pi} \int_{0}^{\pi / 2} e^{-\frac{d_{1} \gamma_{S_{1} D}+d_{2} \gamma_{S_{2} D}+d_{3} \gamma_{R}}{\sin ^{2} \theta}} d \theta\right) \frac{1}{\bar{\gamma}_{S_{1} D} \bar{\gamma}_{S_{2} D} \bar{\gamma}_{R}} e^{-\frac{\gamma_{S_{1} D}}{\bar{\gamma}_{S_{1} D}}-\frac{\gamma_{S_{2} D}}{\bar{\gamma}_{S_{2} D}}-\frac{\gamma_{R}}{\bar{\gamma}_{R}}} d \gamma_{S_{1} D} d \gamma_{S_{2} D} d \gamma_{R} \\
& =\frac{1}{2}\left(1-I\left(d_{1} \bar{\gamma}_{S_{1} D}, d_{2} \bar{\gamma}_{S_{2} D}, d_{3} \bar{\gamma}_{R}\right)-I\left(d_{2} \bar{\gamma}_{S_{2} D}, d_{1} \bar{\gamma}_{S_{1} D}, d_{3} \bar{\gamma}_{R}\right)-I\left(d_{3} \bar{\gamma}_{R}, d_{1} \bar{\gamma}_{S_{1} D}, d_{2} \bar{\gamma}_{S_{2} D}\right)\right) \\
& \stackrel{(*)}{\simeq} \frac{5}{32 d_{1} d_{2} d_{3}} \frac{1}{\bar{\gamma}_{S_{1} D} \bar{\gamma}_{S_{2} D} \bar{\gamma}_{R}} \text {. }
\end{aligned}
$$

it clear to present our analysis, as the main contribution of this paper. BPSK modulation and binary network coding are employed. Channel code CC $[23,35]$ with rate $1 / 2$ is chosen as the one which optimizes both minimum distance and error weight in Rayleigh fading channels [19]. The data packet length is equal to 1024bits. Two network settings are investigated: i) Symmetric: $\bar{\gamma}_{S_{1} D}=\bar{\gamma}_{S_{2} D}=\bar{\gamma}$ and $\bar{\gamma}_{S_{1} R}=$ $\bar{\gamma}_{S_{2} R}=\bar{\gamma}_{R D}=\bar{\gamma}+9 d B$ and ii) Asymmetric: $\bar{\gamma}_{S_{1} D}=\bar{\gamma}$, $\bar{\gamma}_{S_{2} D}=\bar{\gamma}+9$ and $\bar{\gamma}_{S_{1} R}=\bar{\gamma}_{S_{2} R}=\bar{\gamma}_{R D}=\bar{\gamma}+12 d B$. In simulations, $E_{b} N_{0}=\bar{\gamma}-10 \log _{10}(1 / N)$ and it is expended until $25 \mathrm{~dB}$ to clearly see the system diversity order. In addition, the OJNCD algorithms [13] with both DF and DMF are shown for comparison.

Fig. 1 shows analytical and simulation results in the symmetric setting. The first four weights are used in (18). First, it is shown in the figure that the analysis is very close to simulation results. Second, the NO-JNCD gives a performance very close to that of the OJNCD. In this setting, the BERs of both sources are equal.

Fig. 2 presents the results for the asymmetric setting. A similar conclusion is observed: the theoretical analysis curves tightly match the simulations for each source and average BER cases. This proves the accuracy of our analysis.

\section{CONCLUSION}

We proposed a new method that combines the C-MRC detector and the optimal JNCD decoder to analyze the performance of the multiple-access relay channel in quasi-block Rayleigh fading channels. The key idea is to consider the relayed signal as providing additional parity bits (redundancy) of the compound code at the destination. The proposed frame work can be extended to general block fading channels.

\section{REFERENCES}

[1] R. Ahlswede, C. Ning, S. Y. R. Li, and R. W. Yeung, "Network information flow," Information Theory, IEEE Transactions on, vol. 46, no. 4, pp. 1204-1216, 2000.

[2] M. Renzo, L. Iwaza, M. Kieffer, P. Duhamel, and K. Agha, Robust Wireless Network Coding An Overview, vol. 45 of Lecture Notes of the Institute for Computer Sciences, Social Informatics and Telecommunications Engineering, ch. 59, pp. 685-698. Springer Berlin Heidelberg, 2010.

[3] D. H. Woldegebreal and H. Karl, "Multiple-access relay channel with network coding and non-ideal source-relay channels," in Wireless Communication Systems, 2007. ISWCS 2007. 4th International Symposium on, pp. 732-736.
[4] C. Hausl and P. Dupraz, "Joint network-channel coding for the multipleaccess relay channel," in Sensor and Ad Hoc Communications and Networks, 2006. SECON '06. 2006 3rd Annual IEEE Communications Society on, vol. 3, pp. 817-822.

[5] X. Xiaoyan, M. F. Flanagan, N. Goertz, and J. Thompson, "Joint channel and network coding for cooperative diversity in a shared-relay environment," Wireless Communications, IEEE Transactions on, vol. 9, no. 8 , pp. $2420-2423,2010$.

[6] G. Zheng, H. Jie, W. Bing, Z. Shengli, C. Jun-Hong, and P. Willett, "A practical joint network-channel coding scheme for reliable communication in wireless networks," Wireless Communications, IEEE Transactions on, vol. 11, no. 6, pp. 2084-2094, 2012.

[7] P. Weitkemper, D. Wuebben, V. Kuehn, and K.-D. Kammeyer, "Soft information relaying for wireless networks with error- prone source-relay link," in Source and Channel Coding (SCC), 2008 7th International ITG Conference on, pp. 1-6.

[8] K. Lee and L. Hanzo, "Mimo-assisted hard versus soft decodingand-forwarding for network coding aided relaying systems," Wireless Communications, IEEE Transactions on, vol. 8, no. 1, pp. 376-385, 2009.

[9] A. Bletsas, A. Khisti, D. P. Reed, and A. Lippman, "A simple cooperative diversity method based on network path selection," IEEE Journal on Select Areas in Communications, vol. 24, no. 3, pp. 659-672, 2006.

[10] X. Ming and T. Aulin, "Optimal decoding and performance analysis of a noisy channel network with network coding," Communications, IEEE Transactions on, vol. 57, no. 5, pp. 1402-1412, 2009.

[11] C. Deqiang and J. N. Laneman, "Modulation and demodulation for cooperative diversity in wireless systems," Wireless Communications, IEEE Transactions on, vol. 5, no. 7, pp. 1785-1794, 2006. 1536-1276.

[12] T. Wang, A. Cano, G. B. Giannakis, and J. N. Laneman, "Highperformance cooperative demodulation with decode-and-forward relays," IEEE Transactions on Communications, vol. 55, no. 7, pp. 14271438, 2007.

[13] X.-T. Vu, M. Di Renzo, and P. Duhamel, "Optimal and low-complexity iterative joint network/channel decoding for the multiple-access relay channel," in Acoustics, Speech and Signal Processing (ICASSP), 2011 IEEE International Conference on, pp. 3312-3315.

[14] T. Duc and C. Jinho, "Convolutional codes in two-way relay networks with physical-layer network coding," Wireless Communications, IEEE Transactions on, vol. 9, no. 9, pp. 2724-2729, 2010.

[15] R. Knopp and P. A. Humblet, "On coding for block fading channels," Information Theory, IEEE Transactions on, vol. 46, no. 1, pp. 189-205, 2000.

[16] L. Bahl, J. Cocke, F. Jelinek, and J. Raviv, "Optimal decoding of linear codes for minimizing symbol error rate (corresp.)," Information Theory, IEEE Transactions on, vol. 20, no. 2, pp. 284-287, 1974.

[17] A. Glavieux, Channel Coding in Communication Networks: From Theory to Turbo Codes. Wiley\& Sons Ltd, 2007.

[18] M. L. Cedervall and R. Johannesson, "A fast algorithm for computing distance spectrum of convolutional codes," Information Theory, IEEE Transactions on, vol. 35, no. 6, pp. 1146-1159, 1989.

[19] P. Frenger, P. Orten, and T. Ottosson, "Convolutional codes with optimum distance spectrum," Communications Letters, IEEE, vol. 3, no. 11, pp. 317-319, 1999. 